\title{
THE HOMOLOGY AND HIGHER REPRESENTATIONS OF THE AUTOMORPHISM GROUP OF A RIEMANN SURFACE
}

\author{
S. A. BROUGHTON
}

\begin{abstract}
The representations of the automorphism group of a compact Riemann surface on the first homology group and the spaces of $q$-differentials are decomposed into irreducibles. As an application it is shown that $M_{24}$ is not a Hurwitz group.
\end{abstract}

1. Introduction. Let $G$ be a finite group of orientation-preserving homeomorphisms of a Riemann surface $S$ of genus $\sigma \geqslant 2$. We then have a representation of $G$ on the first homology group $H_{1}(S)=H_{1}(S, \mathrm{C})$. If $S$ has a conformal structure which is preserved under the $G$-action, then there are also representations of $G$ on the various spaces of $q$-differentials $\mathscr{H}^{q}(S)\left(\mathscr{H}^{q}(S)=\right.$ holomorphic sections of $T^{*}(S) \otimes \cdots \otimes T^{*}(S)$ ( $q$ times $), T^{*}(S)=$ cotangent bundle). In this note we give formulae (Propositions 1-2) for the decompositions of these representations into irreducibles.

The decompositions for $H_{1}(S) \simeq \mathscr{H}^{1}(S) \oplus \mathscr{H}^{1}(S)^{*}$ and $\mathscr{H}^{2}(S)$ may be applied to the study of surfaces of genus $\sigma$. From the decomposition of the homology representation it follows that the characters of $G$ must satisfy certain inequalities (see (13) below). This is useful in showing that certain groups cannot occur as automorphism groups of a surface of a given genus $\sigma$. In [S] L. L. Scott has given a formula equivalent to (13), though derived by a purely group-theoretic argument.

The decompositions of $\mathscr{H}^{2}(S)$ may be used to locally describe the action of the Teichmüller modular group $\operatorname{Mod}_{\sigma}$ on Teichmüller space, $\mathscr{T}_{\sigma}$ (see $[\mathbf{R}]$ ). This was used by $\mathbf{J}$. Lewittes [L] to compute the dimensions of the branch loci of the action of $\operatorname{Mod}_{\sigma}$ on $\mathscr{T}_{\sigma}$.

The decompositions are derived in $\$ 2$ from the Eichler Trace Formula and the Lefshetz Fixed Point Formula, using a simple character theory argument. In $\$ 3$ we give an application showing that the Mathieu group $M_{24}$ is not a Hurwitz group.

2. The decomposition formulae and their derivations. First we recall some facts about actions of a finite group $G$ on a surface $S$ (cf. $[\mathbf{H}, \mathbf{T}])$. The space $T=S / G$ is a surface $T$ of genus $\tau$, and $\pi: S \rightarrow T$ is branched over $Q_{1}, \ldots, Q_{t} \in T$ with branching orders $n_{1}, \ldots, n_{t}$. Call $\left(\tau: n_{1}, \ldots, n_{t}\right)$ the branching data of $G$ (write $\left(n_{1}, \ldots, n_{t}\right)$ if $\left.\tau=0\right)$. The Riemann-Hurwitz formula [FK, p. 243] gives

$$
(2 \sigma-2) /|G|=2 \tau-2+\sum_{i=1}^{t}\left(1-1 / n_{i}\right) \text {. }
$$

Received by the editors July 23, 1985 and, in revised form, March 21, 1986. 1980 Mathematics Subject Classification (1985 Revision). Primary 30F30. 
We denote the right-hand side by $\kappa$. There are elements $a_{1}, \ldots, a_{\tau}, b_{1}, \ldots, b_{\tau}$, $c_{1}, \ldots, c_{t}$, generating $G$, such that

$$
\prod_{i=1}^{\tau}\left[a_{i}, b_{i}\right] \prod_{j=1}^{t} c_{j}=1,
$$

and

$$
o\left(c_{i}\right)=n_{i} .
$$

If $P \in S$ is a point fixed by $g \in G$, then the induced map of tangent spaces $d g^{-1}$ : $T_{P}(S) \rightarrow T_{P}(S)$ is multiplication by an $o(g)$ th root of unity, denoted by $\varepsilon(P, g)$. It is easy to show that we may pick the $c_{i}$ and $P_{i} \in \pi^{-1}\left(Q_{i}\right)$ such that $G_{P_{i}}=$ $\left\{g \in G \mid g P_{i}=P_{i}\right\}=\left\langle c_{i}\right\rangle$ and

$$
\varepsilon\left(P_{i}, c_{i}\right)=\exp \left(2 \pi \sqrt{-1} / n_{i}\right) .
$$

Let $U_{n} \subseteq S^{1}$ be the group of $n$th roots of unity and let $\varphi_{k}: S^{1} \rightarrow S^{1}$ be the character $z \rightarrow z^{k}, k \in \mathbf{Z}$. Let $c_{1}, \ldots, c_{t}$ be as defined above and let $\nu_{i}:\left\langle c_{i}\right\rangle \rightarrow U_{n_{i}}$ be the isomorphism defined by $c_{i} \rightarrow \exp \left(2 \pi \sqrt{-1} / n_{i}\right)$. Let $\chi_{0}, \ldots, \chi_{l}$ be the irreducible characters of $G$ with $\chi_{0}=$ principal character. Each $\chi_{j}$ defines a character of $U_{n_{i}}$ by means of the isomorphism $\nu_{i}$. Define $m_{i}^{k}\left(\chi_{j}\right), 0 \leqslant k \leqslant n_{i}-1$, by

$$
\chi_{j}\left|U_{n_{i}}=\sum_{k=0}^{n_{i}-1} m_{i}^{k}\left(\chi_{j}\right) \varphi_{k}\right| U_{n_{i}}
$$

and define $m_{i}^{k}\left(\chi_{j}\right)$ for all $k \in \mathbf{Z}$ by periodicity: $m_{i}^{k}\left(\chi_{j}\right)=m_{i}^{k+n_{i}}\left(\chi_{j}\right)$. Let $\operatorname{ch}_{\mathscr{C}^{q}(S)}$ be the character of the representation of $G$ on $\mathscr{H}^{q}(S)$, and write

$$
\operatorname{ch}_{\mathscr{H}(S)}=\mu_{q}^{0} \chi_{0}+\cdots+\mu_{q}^{l} \chi_{l} .
$$

Define the Poincare series $P_{\chi_{j}}(z)$ by

$$
P_{\chi_{j}}(z)=\sum_{q=0}^{\infty} \mu_{q}^{j} z^{q}
$$

We have the following propositions.

PROPOSITION 1. Let $G$ be a group of conformal automorphisms of a Riemann surface $S$ of genus $\geqslant 2$ and let all notation be as above. Then:

(i) $P_{\chi_{0}}(z)=1+z+z R_{\chi_{0}}(z)$,

(ii) $P_{\chi_{j}}=z R_{\chi_{j}}(z), j \neq 0$, where

(iii)

$$
R_{\chi_{j}}=\frac{(1-\tau) \chi_{j}(1)}{1-z}+\frac{\kappa \chi_{j}(1)}{(1-z)^{2}}-\sum_{i=1}^{t} \frac{1}{n_{i}} \cdot \frac{e_{i}^{0}(j)+e_{i}^{1}(j) z+\cdots+e_{i}^{n_{i}-1}(j) z^{n_{i}-1}}{1-z^{n_{i}}}
$$

and

$$
e_{i}^{r}(j)=\sum_{k=0}^{n_{i}-1} k \cdot m_{i}^{1+r+k}\left(\chi_{j}\right)
$$


Proposition 2. Let $G$ be a finite group of homeomorphisms of a Riemann surface $S$, $\mathrm{ch}_{H_{1}(S)}$ the character of the homology representation, and other notation as above. Then the multiplicity of $\chi_{j}$ in $\mathrm{ch}_{H_{1}(S)},\left\langle\chi_{j}, \mathrm{ch}_{H_{1}(S)}\right\rangle$, is given by

(i) $\left\langle\chi_{0}, \mathrm{ch}_{H_{1}(S)}\right\rangle=2 \tau$,

(ii)

$$
\left\langle\chi_{j}, \operatorname{ch}_{H_{1}(S)}\right\rangle=(2 \tau-2+t) \chi_{j}(1)-\sum_{i=1}^{t} m_{i}^{0}\left(\chi_{j}\right), \quad j \neq 0 .
$$

Let $\rho$ be the regular representation of $G$ and $\rho_{i}$ the permutation character determined by $G$ acting on the coset space $G /\left\langle c_{i}\right\rangle$. Then (i) and (iii) may be rewritten:

(iii)

$$
\operatorname{ch}_{H_{1}(S)}=2 \chi_{0}+(2 \tau-2+t) \rho-\sum_{i=1}^{t} \rho_{i} .
$$

Before proving Propositions 1-2 we recall the Eichler Trace Formula and the Lefschetz Fixed Point Formula. Let $\eta: G \rightarrow \mathbf{Z}$ be the class function on $G$ obtained by setting $\eta(g)$ equal to the negative of the Euler characteristic of the fixed point subset $S^{g}$ of $g$, i.e.

$$
\eta(1)=2 \sigma-2, \quad \eta(g)=-\left|S^{g}\right|, \quad g \neq 1 .
$$

By the Lefschetz Fixed Point Formula,

$$
\operatorname{ch}_{H_{1}(S)}(g)=2+\eta(g), \quad g \in G .
$$

Define $\lambda_{q}: G \rightarrow \mathbf{C}, q \geqslant 0$, as follows:

$$
\begin{aligned}
& \lambda_{0}(g)=1, \quad g \in G, \\
& \lambda_{q}(1)=(\sigma-1)(2 q-1), \quad q \geqslant 1, \\
& \lambda_{q}(g)=\sum_{P \in S^{g}} \frac{(\varepsilon(P, g))^{q}}{1-\varepsilon(P, g)}, \quad q \geqslant 1,
\end{aligned}
$$

where the last sum is zero if $S^{g}$ is empty. The Riemann-Roch Theorem and the Eichler Trace Formula state that the characters $\operatorname{ch}_{\mathscr{H}^{q}(S)}$ are given by

$$
\begin{aligned}
& \operatorname{ch}_{\mathscr{H}^{q}(S)}(g)=\lambda_{q}(g), \quad q \neq 1, \\
& \operatorname{ch}_{\mathscr{H}^{1}(S)}(g)=1+\lambda_{q}(g) .
\end{aligned}
$$

For proofs of (6)-(7) see [FK]. Observe $[\mathbf{F K}]$ that $\eta(g)=2 \operatorname{Re} \lambda_{1}(g)$.

Write

$$
\eta=\eta^{0}+\cdots+\eta^{t}, \quad \lambda_{q}=\lambda_{q}^{0}+\cdots+\lambda_{q}^{t},
$$

where

$$
\begin{aligned}
& \eta^{0}(1)=2 \sigma-2, \quad \eta^{0}(g)=0, \quad g \neq 1, \\
& \eta^{i}(1)=0, \quad \eta^{i}(g)=-\left|S^{g} \cap \pi^{-1}\left(Q_{i}\right)\right|, \quad i>0, \\
& \lambda_{q}^{0}(1)=(\sigma-1)(2 q-1), \quad \lambda_{q}^{0}(g)=0, \quad g \neq 1, q \geqslant 1, \\
& \lambda_{q}^{i}(1)=0, \quad \lambda_{q}^{i}(g)=\sum_{P \in S^{g} \cap \pi^{-1}\left(Q_{i}\right)} \frac{(\varepsilon(P, g))^{q}}{1-\varepsilon(P, g)}, \quad i>0, q \geqslant 1 .
\end{aligned}
$$


For $1 \neq g \in G, S^{g} \cap \pi^{-1}\left(Q_{i}\right) \neq \varnothing$ if and only if the conjugacy class of $g, \mathrm{Cl}(g)$, meets $\left\langle c_{i}\right\rangle$. Asume $g \in\left\langle c_{i}\right\rangle$, then since $G_{P_{i}}$ is cyclic, $S^{g} \cap \pi^{-1}\left(Q_{i}\right)$ is in 1-1 correspondence with $N_{G}(\langle g\rangle) /\left\langle c_{i}\right\rangle$ by $h \rightarrow h \cdot P_{i}$. Furthermore, $N_{G}(\langle g\rangle) / \operatorname{Cent}(g)$ is in 1-1 correspondence wih $\mathrm{Cl}(g) \cap\left\langle c_{i}\right\rangle$ by $h \rightarrow h g h^{-1}$. From (4) and the definition of $\nu_{i}, \varepsilon\left(P_{i}, g\right)=\nu_{i}(g), g \in\left\langle c_{i}\right\rangle$. It easily follows for $i>0$ that

$$
\lambda_{q}^{i}(g)=\frac{|\operatorname{Cent}(g)|}{n_{i}} \cdot \sum_{h \in \mathrm{Cl}(g) \cap\left\langle c_{i}\right\rangle} \frac{\left(\nu_{i}(h)\right)^{q}}{1-\nu_{i}(h)} .
$$

Since $\lambda_{q}^{i}$ is a class function, this holds for all $1 \neq g \in G$. Similarly, for $1 \neq g \in G$,

$$
\eta^{i}(g)=-\frac{|\operatorname{Cent}(g)|}{n_{i}}\left|\mathrm{Cl}(g) \cap\left\langle c_{i}\right\rangle\right| .
$$

We now give proofs of the decompositions, first Proposition 2. Let $1=g_{0}, \ldots, g_{\text {l }}$ be a set of representatives of conjugacy classes of $G$. For $j=0,1, \ldots, l$ :

$$
\begin{aligned}
\left\langle\eta, \chi_{j}\right\rangle & =\sum_{i=0}^{t}\left\langle\eta^{i}, \chi_{j}\right\rangle=\sum_{i=0}^{t} \frac{1}{|G|} \sum_{g \in G} \eta^{i}(g) \bar{\chi}_{j}(g) \\
& =\sum_{i=0}^{t} \sum_{k=0}^{l} \frac{\eta^{i}\left(g_{k}\right) \bar{\chi}_{j}\left(g_{k}\right)}{\left|\operatorname{Cent}\left(g_{k}\right)\right|} \\
& =\frac{2 \sigma-2}{|G|} \chi_{j}(1)-\sum_{i=1}^{t} \frac{1}{n_{i}} \sum_{1 \neq g \in\left\langle c_{i}\right\rangle} \overline{\chi_{j}(g)},
\end{aligned}
$$

from (9) above. By the Riemann-Hurwitz Formula (1), (10) may be rewritten as

$$
(2 \tau-2+t) \chi_{j}(1)-\sum_{i=1}^{t} \frac{1}{n_{i}} \sum_{g \in\left\langle c_{i}\right\rangle} \bar{\chi}_{j}(g)=(2 \tau-2+t) \chi_{j}(1)-\sum_{i=1}^{t} m_{i}^{0}\left(\chi_{j}\right) .
$$

Since $\operatorname{ch}_{H_{1}(S)}=2 \chi_{0}+\eta$, (i) and (ii) of Proposition 2 follow immediately; (iii) follows from (i)-(ii) and Frobenius reciprocity.

Let $R_{g}(z)=\sum_{q=1}^{\infty} \lambda_{q}(g) z^{q-1}$. To prove Proposition 1 it suffices by (7) to prove

$$
R_{\chi_{j}}(z)=\frac{1}{|G|} \sum_{g \in G} R_{g}(z) \overline{\chi_{j}(g)} .
$$

Using (8) and arguing as above, the right-hand side of (11) equals

$$
\begin{aligned}
\sum_{q=1}^{\infty} \frac{(\sigma-1) \chi_{j}(1)}{|G|}(2 q-1) z^{q-1}+\sum_{i=1}^{t} \sum_{q=1}^{\infty} \sum_{1 \neq g \in\left\langle c_{i}\right\rangle} \frac{1}{n_{i}} \frac{\left(\nu_{i}(g)\right)^{q}}{1-\nu_{i}(g)} \bar{\chi}_{j}(g) z^{q-1} \\
=\kappa \chi_{j}(1)(1-z)^{-2} \frac{\kappa \chi_{j}(1)(1-z)^{-1}}{2} \\
\quad+\sum_{i=1}^{t} \sum_{r=0}^{n_{i}-1} \frac{1}{n_{i}} \sum_{1 \neq \omega \in U_{n_{i}}} \frac{\omega^{r+1}}{1-\omega} \bar{\chi}_{j}(\omega) z^{r}(1-z)^{-n_{i}} .
\end{aligned}
$$


We calculate

$$
\begin{aligned}
\sum_{1 \neq \omega \in U_{n}} \frac{\omega^{s}}{1-\omega} \bar{\chi}(\omega) & =\lim _{x \rightarrow 1} \sum_{1 \neq \omega \in U_{n}} \frac{\omega^{s}}{1-x \omega} \bar{\chi}(\omega) \\
& =\lim _{x \rightarrow 1}\left(\sum_{q=0}^{\infty} \sum_{\omega \in U_{n}} \omega^{q+s} \bar{\chi}(\omega) x^{q}-\sum_{q=0}^{\infty} \chi(1) x^{q}\right) \\
& =\lim _{x \rightarrow 1}\left(n \frac{L_{s}(x)}{1-x^{n}}-\frac{\chi(1)}{1-x}\right),
\end{aligned}
$$

where $L_{s}(x)=a_{0}+a_{1} x+\cdots+a_{n-1} x^{n-1}$ and $a_{k}=(1 / n) \sum_{\omega \in U_{n}} \omega^{k+s} \bar{\chi}(\omega)$. The limit is easily calculated by l'Hôpital's rule and equals $(n-1) \chi(1) / 2-L_{s}^{\prime}(1)$. Setting $n=n_{i}, s=r+1, \chi=\chi_{j}$, then $a_{k}=m_{i}^{1+k+r}\left(\chi_{j}\right)$ and (11) now follows easily from (12) and the definition of $R_{\chi_{j}}$.

3. Application. If $\tau=0$, then $G$ is generated by $c_{1}, \ldots, c_{t}$ with $c_{1} \cdot c_{2} \cdots c_{t}=1$, and from (ii) of Proposition 2 it follows that for a nonprincipal character $\chi_{j}$

$$
(t-2) \chi_{j}(1) \geqslant \sum_{i=1}^{t} m_{i}^{0}\left(\chi_{j}\right) \text {. }
$$

This is a reformulation of the inequality that L. L. Scott obtains in [S] by purely group theoretic means for arbitrary characteristic. The $G$-module he constructs on p. 475 of [S] may be identified with $H_{1}(S)$. The inequality (13) may sometimes be used as a "Brauer trick" to show that a given group cannot occur as the automorphism group of a surface of given genus.

As an example of this let us verify that the Mathieu group $M_{24}$ is not a Hurwitz group. The group $G$ is a Hurwitz group if it occurs as the automorphism group of a surface $S$ of genus $\sigma$ with $|G|=84(\sigma-1)$, Hurwitz' upper bound for the order of an automorphism group. If $G$ acts on $S$ as above then the branching data is $(2,3,7)$ and $G$ has a generating $(2,3,7)$-vector $\left(c_{1}, c_{2}, c_{3}\right)$. In Table 1 we have copied a portion of the character table of $M_{24}$ [Fr, p. 346], giving, for selected characters, the character values of all elements of order $1,2,3$, or 7 . The classes are given in cycle notation, $M_{24}$ being realized as a permutation group of degree 24 .

TABle 1

\begin{tabular}{lrrrrrcc} 
& $1^{24}$ & $1^{8} 2^{8}$ & $2^{12}$ & $1^{6} 3^{6}$ & $3^{8}$ & $1^{3} 7_{+}^{3}$ & $1^{3} 7_{-}^{3}$ \\
\cline { 2 - 7 }$\chi_{1}$ & 45 & -3 & 5 & 0 & 3 & $(-1+\sqrt{-7}) / 2$ & $(-1-\sqrt{-7}) / 2$ \\
$\chi_{2}$ & 252 & 28 & 12 & 9 & 0 & 0 & 0
\end{tabular}

For $c_{i}$ chosen from the classes in Table 1 all the nonidentity elements of $\left\langle c_{i}\right\rangle$ are conjugate in $M_{24}$ except for $\left\langle c_{3}\right\rangle$, where half lie in $1^{3} 7_{+}^{3}$ and the other half lie in $1^{3} 7_{-}^{3}$. Since $\kappa=1 / 42$, we obtain from (10), for any nonprincipal character $\chi$ of $M_{24}$,

$$
\frac{1}{42}\left(\chi(1)-21 \chi\left(c_{1}\right)-28 \chi\left(c_{2}\right)-36 \operatorname{Re} \chi\left(c_{3}\right)\right)=\langle\eta, \chi\rangle \geqslant 0
$$

or

$$
\chi(1) \geqslant 21 \chi\left(c_{1}\right)+28 \chi\left(c_{2}\right)+36 \operatorname{Re} \chi\left(c_{3}\right)
$$


(This is equivalent to (13) but slightly more convenient.) There is no possible choice of $c_{1}, c_{2}, c_{3}$ for which this inequality holds for both the characters $\chi_{1}, \chi_{2}$ above. It is interesting to note that for $c_{1} \in 2^{12}, c_{2} \in 3^{8}, c_{3} \in 1^{3} 7_{+}^{3}$, or $1^{3} 7_{-}^{3}, \chi_{2}$ and its conjugate $\bar{\chi}_{2}$ are the only irreducible characters for which (13) fails, and that the standard Brauer trick [I, p. 70] applied to any pair of $\left\langle c_{1}\right\rangle,\left\langle c_{2}\right\rangle$, or $\left\langle c_{3}\right\rangle$ fails.

\section{REFERENCES}

[FK] H. M. Farkas and I. Kra, Riemann surfaces, Graduate Texts in Math., no. 71, Springer-Verlag, Berlin and New York, 1979.

[Fr] F. G. Frobenius, Gesammelte Abhandlungen, Vol. 3, Springer-Verlag, Berlin and New York, 1968.

[H] J. Harvey, On branch loci in Teichmüller space, Trans. Amer. Math. Soc. 153 (1971), 387-399.

[I] I. M. Isaacs, Character theory of finite groups, Academic Press, New York, 1976.

[L] J. Lewittes, Invariant quadratic differentials, Bull. Amer. Math. Soc. 68 (1962), 320-322.

[R] H. E. Rauch, A transcendental view of the space of algebraic Riemann surfaces, Bull. Amer. Math. Soc. 21 (1965), 1-39.

[S] L. L. Scott, Matrices and cohomology, Ann. of Math. (2) 105 (1977), 473-492.

[T] T. Tucker, Finite groups acting on surfaces and the genus of a group. J. Combin. Theory Ser. B 34 (1983), 82-98.

Department of Mathematics, University of Wisconsin - Madison, Madison, Wisconsin 53706

Current address: Department of Mathematics, Cleveland State University, Cleveland, Ohio 44115 